\title{
The anatomy and isometry of a quasi- anatomical reconstruction of the medial patellofemoral ligament
}

DanielPérez-Prieto 1,*Phone+34-932483196Emaildperezprieto@hospitaldelmar.cat BrunoCapurro 1

PabloE.Gelber 2

GerardGinovart 3

FranciscoReina 4

VicenteSanchis-Alfonso 5

JoanC.Monllau 1

1Department of Orthopaedic Surgery and Traumatology, Hospital del

MarUniversitat Autònoma de BarcelonaPasseig Marítim, 25BarcelonaSpain

2Orthopedic DepartmentHospital de la Santa Creu i Sant PauBarcelonaSpain

3Hospital Terres de l'EbreTortosaSpain

4Faculty of MedicineUniversitat de GironaGironaSpain

5Hospital 9 de OctubreValenciaSpain

\section{Abstract}

\section{Purpose}

To describe the anatomy of the medial patellofemoral ligament (MPFL) and its relationship to the Adductor Magnus (AM) tendon as well as the behaviour exhibited in length changes during knee flexion.

\section{A01 \\ A02 \\ Methods}

Ten cadaveric knees were dissected. The length from the superior and inferior patellar origin of the MPFL to its femoral insertion was measured at different degrees of knee flexion $\left(0^{\circ}, 30^{\circ}, 60^{\circ}, 90^{\circ}\right.$ and $\left.120^{\circ}\right)$. The same measures were made from both patellar origins of the MPFL up to the femoral insertion of the AM. The distance between the insertion of the AM and the Hunter canal was also measured.

\section{$\underline{\mathrm{AO3}}$}

\section{Results}

In general, isometry up to $90^{\circ}$ was seen in all measures of the MPFL and those of the AM. The most isometric behaviour was seen in 2 measures: the length of the AM femoral insertion up to the inferior origin of the MPFL on the patella and the length of the femoral insertion of the MPFL up to the inferior origin of the MPFL on the patella. Similar behaviour was seen regardless of the anatomical or quasianatomical femoral point of attachment (n.s.). The distance from the AM tendon to the Hunter canal had a mean value of $78.6 \mathrm{~mm}$ (SD $9.4 \mathrm{~mm}$ ). 


\section{Conclusion}

The behaviour exhibited during the changes in the length of the anatomical femoral footprint of the MPFL and the AM is similar. Neurovascular structures were not seen at risk. This is relevant in the daily clinical practice since the AM tendon might be a suitable point of insertion for MPFL reconstruction.

Keywords

Medial patellofemoral ligament

Femoral attachment

Quasi-anatomical reconstruction

Patellofemoral instability

\section{Introduction}

The medial patellofemoral ligament (MPFL) is the main restrictor of lateral dislocation of the patellofemoral joint $[7,14,19,22]$. It is usually torn in cases of objective patellofemoral instability, and therefore its reconstruction is crucial to the treatment of the aforementioned pathology [4, 17].

Knowing the biomechanical performance of the MPFL is essential to carrying out a correct surgical technique $[1,2,5]$. Several researchers have studied the anatomy of the MPFL and its behaviour at different degrees of knee flexion [6, 18, 21]. Recently, a new non-anatomical technique in which the Adductor Magnus (AM) tendon was used as a pulley instead of the classical femoral attachment for MPFL reconstruction has been reported [9]. Although the authors reported excellent outcomes in patients treated with that procedure, the anatomical relationships of this type of MPFL reconstruction as well as its behaviour during knee motion have not been thoroughly analysed.

The purpose of this study was to describe the anatomy of the MPFL and AM. First, the functional length of the MPFL during knee flexion in cadaveric knees was measured. Then, the functional distance between the AM femoral insertion and the patellar insertion of the MPFL (as it is described in the aforementioned nonanatomical reconstruction technique [9]) was also calculated. The behaviour of both measures during flexion was then compared. The hypothesis was that the nonanatomical AM attachment behaves, biomechanically speaking, similarly to the anatomical femoral insertion of the MPFL. This might be of clinical interest to use the AM tendon as a pulley in the reconstruction of the MPFL.

\section{Materials and methods}

Ten fresh-frozen cadaveric knees (whole leg specimen) from 7 cadavers were dissected. Seven knees were from male cadavers and 3 were from female cadavers, all of which corresponded to elderly people and the ages ranged from 59 to 74 years 
old. None of them had reported any history of rheumatic disease. Neither had the included knees undergone any previous surgery.

The first part of this study consisted in defining the anatomy of the AM and the MPFL. After the dissection and removal of the broad skin and soft tissue window of the anteromedial aspect of the joint, a blunt dissection was made to identify the triangular/trapezoidal shape of the MPFL. The AM was also dissected from its attachment up to the Hunter canal. Three points were identified. They were the MPFL femoral attachment (MPFL-F), its superior origin on the patella (MPFL-SP) and its inferior origin on the patella (MPFL-IP). Subsequently, the femoral insertion of the AM (AM-F) was also identified. All points were identified following the LaPrade anatomical studies [7].

During the second part of the study, isometry was evaluated at 5 fixed angles of knee flexion $\left(0^{\circ}, 30^{\circ}, 60^{\circ}, 90^{\circ}\right.$ and $\left.120^{\circ}\right)$. Four measures were taken at each degree of motion (Fig. 1): the distance between MPFL-F and MPFL-SP, the distance between MPFL-F and MPFL-IP, the distance between the AM-F and MPFL-SP and the distance between AM-F and MPFL-IP. Some other measures such as the distance between the insertion of the AM and the Hunter canal were included.

\section{Fig. 1}

Detail of one specimen that shows the anatomical landmarks. Arrow femoral insertion of the medial patellofemoral ligament. Head arrow femoral insertion of the adductor magnus. Solid ring superior patellar origin of the patellofemoral ligament. Outlined ring inferior patellar origin of the patellofemoral ligament

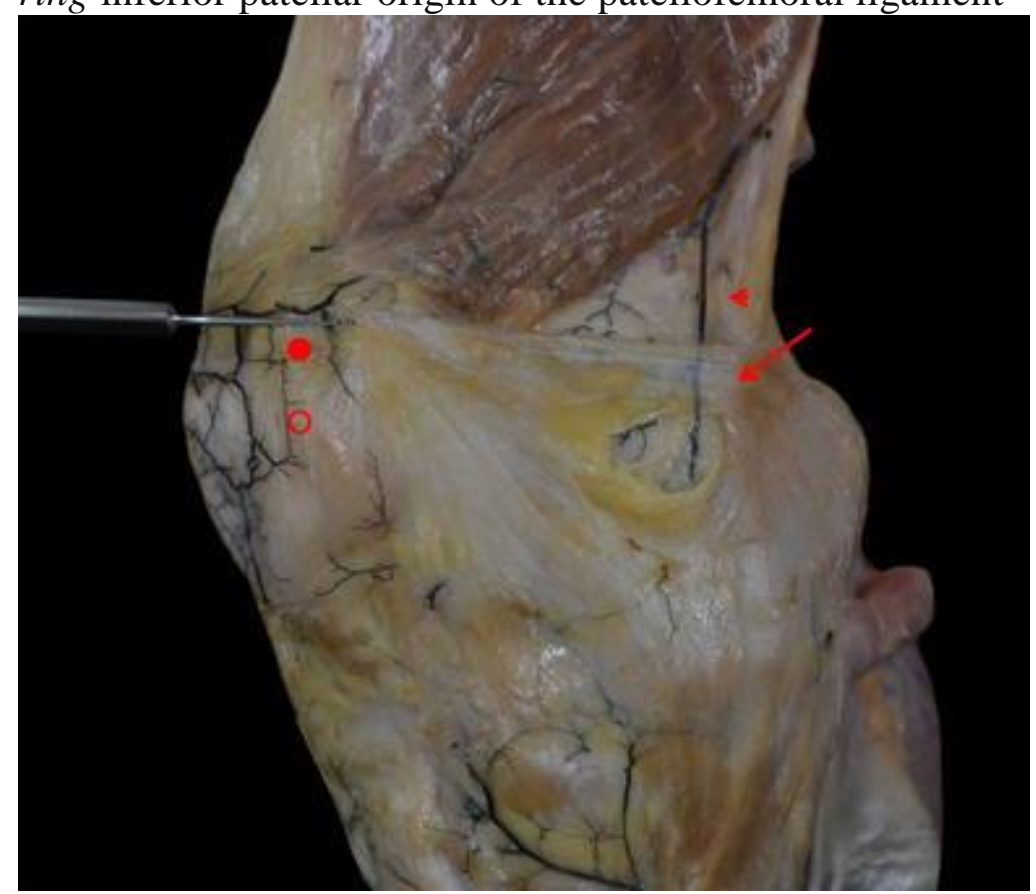

Measures were made using a digital calliper (Digimatic Caliper, Mitutoyo, Japan; 0.01 accuracy) and a digital goniometer (Absolute Goniometer; Fabrication Enterprises Inc., White Plains, NY, USA; $0.5^{\circ}$ accuracy). All measurements were obtained by the same individual so as to avoid interobserver error. The distances 
between all the points were measured at least twice so as to minimize intraobserver error and were then rounded to the nearest decimal of millimetre.

\section{IRB approval}

The study was approved by the local Ethics Committee (ICATME-Institut Universitari Dexeus, 3/2014).

\section{Statistical analysis}

Continuous variables are expressed as mean and standard deviations (SD). When 2 related items of data were analysed, the Student's $t$ test was used. In all cases, a $p$ value of $<0.05$ was considered statistically significant. The statistical analysis was done using the SPSS 18.0 (SPSS Inc., Chicago, IL, USA) statistical package. No sample size analysis was made because of the descriptive aim of this study and also because the specimens were limited in number.

\section{Results}

The distance from the AM tendon attachment to the Hunter canal had a mean value of $78.6 \mathrm{~mm}$ (SD $9.4 \mathrm{~mm}$ ).

The mean distance from the femoral attachment of the MPFL up to its superior patellar origin was $57.8 \mathrm{~mm}$ (SD $6.4 \mathrm{~mm}$ ), while the mean distance up to its inferior patellar origin was $55.7 \mathrm{~mm}$ (SD $6.6 \mathrm{~mm}$ ). The distances from the quasi-anatomical attachment, which were described by Monllau et al. [9], up to the anatomical origin of the MPFL were also measured. In that sense, the mean distance from the femoral insertion of the AM up to the superior patellar origin of the MPFL was $60.7 \mathrm{~mm}$ (SD $5.7 \mathrm{~mm}$ ), while the mean distance up to the inferior patellar origin of the MPFL was $59.5 \mathrm{~mm}$ (SD $6.1 \mathrm{~mm})$.

According to Smirk and Morris [18], all MPFL measures and those from the AM showed isometry up to $90^{\circ}$. The most isometric behaviour was seen in the measure between the AM femoral insertion and femoral insertion of the MPFL up to the inferior origin of the MPFL on the patella. Therefore, similar performance (n.s.) might be expected of those two bundles, as can be seen in Fig. 2. The measures from the superior origin of the MPFL showed a small decrease from $0^{\circ}$ to $30^{\circ}$ and a bigger drop from $90^{\circ}$ to $120^{\circ}$. However, similar behaviour (n.s.) was seen regardless of the anatomical or quasi-anatomical femoral point, as can be seen in Fig. 3.

\section{Fig. 2}

Length changes of superior bundles. Results are expressed as mean and standard deviation (in parentheses) 


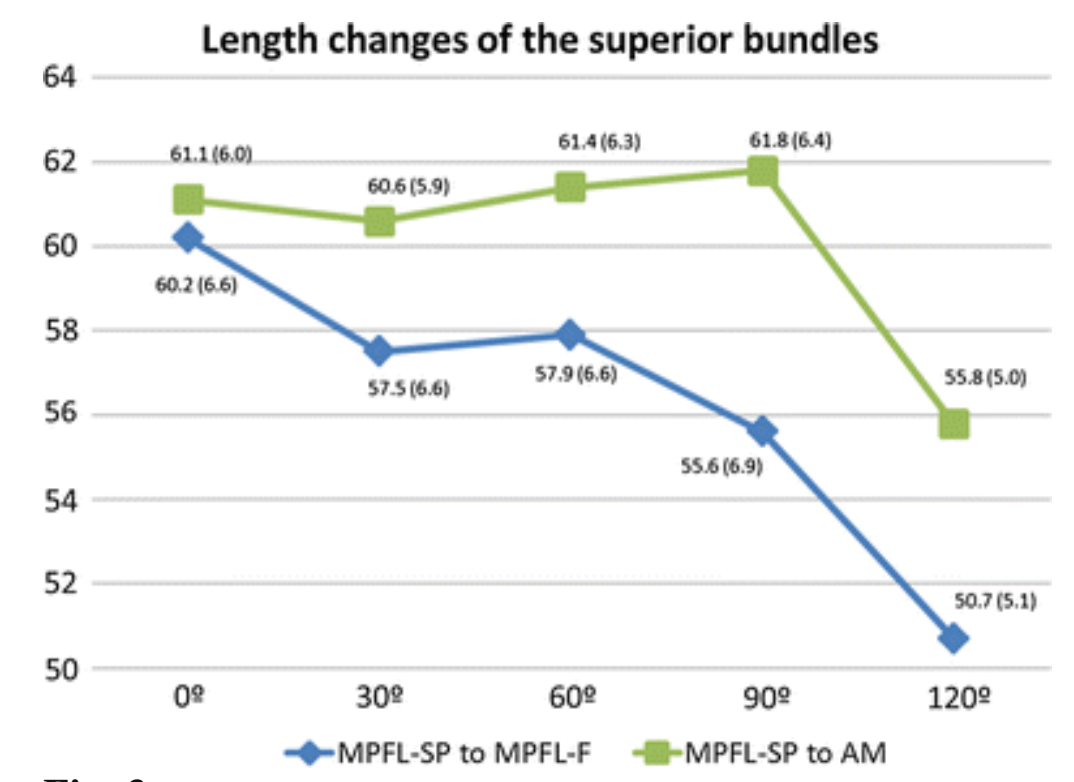

Fig. 3

Length changes of inferior bundles. Results are expressed as mean and standard deviation (in parentheses)

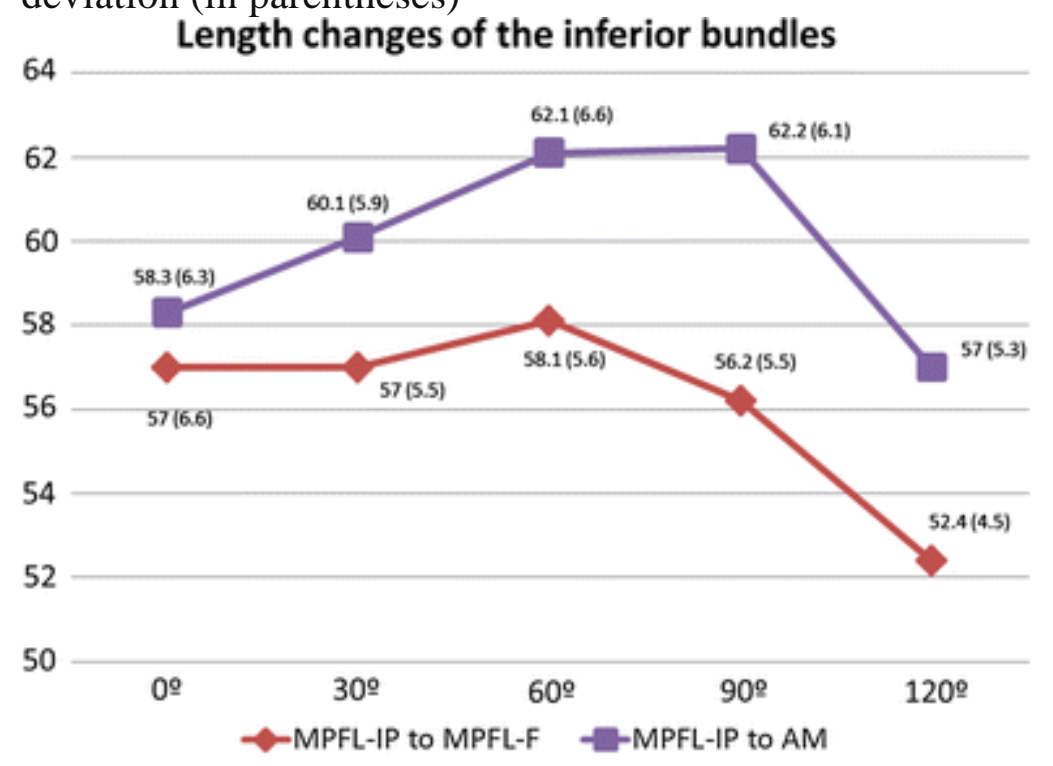

\section{Discussion}

The most important finding of the present study was that the non-anatomical AM attachment has biomechanical behaviour similar to the anatomical femoral insertion of the MPFL. In that sense, the hypothesis was confirmed. As secondary findings, the isometry of the MPFL has been demonstrated to range from $0^{\circ}$ to $90^{\circ}$, most importantly in the fibres that originate in the inferior part of the patella. There is substantive controversy with respect to the anatomy of the MPFL [25]. There are authors who have described it as a continuation of the vastus medialis obliquus, while others see it as an independent structure [1, 2, 11, 14, 18, 21]. It has also been described as both a single bundle ligament and as a complex ligament with different attachments at the patella and femur [8, 15]. In terms of isometry, there is also significant debate $[3,10,12,23]$. Nonetheless, taking a difference of 
less than $5 \mathrm{~mm}$ as an isometric ligament (as proposed by Smirk [18]), the present results showed isometry between $0^{\circ}$ and $90^{\circ}$. However, the superior fibres of the ligament may exhibit biphasic behaviour from $0^{\circ}$ to $30^{\circ}$ and then from $30^{\circ}$ to $90^{\circ}$. The aforementioned anatomical studies have been used to suggest different surgical techniques to treat MPFL tears. There are several authors who propose an anatomical reconstruction of the MPFL [17, 20], even using minimally invasive techniques [26]. They state that finding the femoral insertion of the MPFL is crucial to achieving good outcomes. Although they report positive results using anatomical techniques, there is no prospective randomized study that compares anatomical and non-anatomical surgical techniques for MPFL reconstruction. However, there are in vivo anatomical studies that report on the superior behaviour of a non-anatomical reconstruction of the MPFL [13, 25]. Similarly, good results have been described with non-anatomical (or quasi-anatomical) techniques [9, 16]. Monllau et al. [9] recently reported an improvement in functional scores using their technique, with no recurrence at a minimum 27 month follow-up. In that study, a quasi-anatomical reconstruction of the MPFL was performed with the AM tendon as a pulley.

Although the anatomy of the AM insertion into the distal femur and its relationship with the MPFL femoral insertion have been defined by LaPrade et al. [7, 24], their biomechanics and the functional length during flexion haven not been well defined. The results found in the present study show similar behaviour in the distances between the patellar insertion of the MPFL and both its insertion in the femur and the AM femoral insertion. Moreover, a mean distance from the AM insertion up to the Hunter canal was found to be some $78 \mathrm{~mm}$, enough to avoid damage to the neurovascular structures.

Several limitations can be attributed to the present study. First, and most important, the cadavers used were all elderly. Neither an arthrotomy nor radiographs were performed to assess the presence of patellofemoral arthrosis. Additionally, there was a lack of information about any previous patellofemoral instability. Second, soft tissues of the cadavers can be damaged, but not those in young in vivo patients. An additional limitation is the fact that linear measures were taken in the cadavers, whereas the in vivo MPFL has a somewhat curved shape. The last limitation is the small number of specimens used in the present study.

\section{Conclusion}

In conclusion, similar biomechanical behaviour has been found in the anatomy of the MPFL and the quasi-anatomical reconstruction of the MPFL using the AM femoral insertion. Both of them showed isometry from $0^{\circ}$ to $90^{\circ}$, most importantly the fibres that originate in the inferior part of the patella.

\section{References}

1. 
Amis AA, Firer P, Mountney J, Senavongse W, Thomas NP (2003) Anatomy and biomechanics of the medial patellofemoral ligament. Knee 10(3):215-220

2.

Conlan T, Garth WP Jr, Lemons JE (1993) Evaluation of the medial soft-tissue restraints of the extensor mechanism of the knee. J Bone Joint Surg Am 75(5):682693

3.

Cossey AJ, Paterson R (2005) A new technique for reconstructing the medial patellofemoral ligament. Knee 12(2):93-98

4.

Dejour H, Walch G, Nove-Josserand L, Guier C (1994) Factors of patellar instability: an anatomic radiographic study. Knee Surg Sports Traumatol Arthrosc 2(1):19-26

5.

Desio SM, Burks RT, Bachus KN (1998) Soft tissue restraints to lateral patellar translation in the human knee. Am J Sports Med 26(1):59-65 6.

Graf M, Diether S, Vlachopoulos L, Fucentese S, Furnstahl P (2014) Automatic string generation for estimating in vivo length changes of the medial patellofemoral ligament during knee flexion. Med Biol Eng Comput 52(6):511-520

7.

LaPrade MD, Kennedy MI, Wijdicks CA, LaPrade RF (2015) Anatomy and biomechanics of the medial side of the knee and their surgical implications. Sports Med Arthrosc 23(2):63-70

8.

Mochizuki T, Nimura A, Tateishi T, Yamaguchi K, Muneta T, Akita K (2013) Anatomic study of the attachment of the medial patellofemoral ligament and its characteristic relationships to the vastus intermedius. Knee Surg Sports Traumatol Arthrosc 21(2):305-310

9.

Monllau JC, Pelfort X, Gelber P, Tey M (2013) Combined tibial tubercle realignment and MPFL reconstruction. In: Sanchis-Alfonso V (ed) Patellar instability surgery in clinical practice, chapter 28, 2nd edn. Springer, London, pp $39-85$ 
Mountney J, Senavongse W, Amis AA, Thomas NP (2005) Tensile strength of the medial patellofemoral ligament before and after repair or reconstruction. J Bone Joint Surg Br 87(1):36-40

11.

Nomura E, Inoue M, Osada N (2005) Anatomical analysis of the medial patellofemoral ligament of the knee, especially the femoral attachment. Knee Surg Sports Traumatol Arthrosc 13(7):510-515

12.

Ostermeier S, Holst M, Bohnsack M, Hurschler C, Stukenborg-Colsman C, Wirth CJ (2007) In vitro measurement of patellar kinematics following reconstruction of the medial patellofemoral ligament. Knee Surg Sports Traumatol Arthrosc 15(3):276-285

13.

Panagopoulos A, van Niekerk L, Triantafillopoulos IK (2008) MPFL reconstruction for recurrent patella dislocation: a new surgical technique and review of the literature. Int J Sports Med 29(5):359-365

14.

Philippot R, Chouteau J, Wegrzyn J, Testa R, Fessy MH, Moyen B (2009) Medial patellofemoral ligament anatomy: implications for its surgical reconstruction. Knee Surg Sports Traumatol Arthrosc 17(5):475-479

15.

Placella G, Tei MM, Sebastiani E, Criscenti G, Speziali A, Mazzola C, Georgoulis A, Cerulli G (2014) Shape and size of the medial patellofemoral ligament for the best surgical reconstruction: a human cadaveric study. Knee Surg Sports Traumatol Arthrosc 22(10):2327-2333

16.

Sanchis-Alfonso V, Ramirez-Fuentes C, Montesinos-Berry E, Aparisi-Rodriguez F, Marti-Bonmati L (2015) Does radiographic location ensure precise anatomic location of the femoral fixation site in medial patellofemoral ligament surgery? Knee Surg Sports Traumatol Arthrosc. doi:10.1007/s00167-015-3523-x

17.

Schottle PB, Fucentese SF, Romero J (2005) Clinical and radiological outcome of medial patellofemoral ligament reconstruction with a semitendinosus autograft for patella instability. Knee Surg Sports Traumatol Arthrosc 13(7):516-521

18.

Smirk C, Morris H (2003) The anatomy and reconstruction of the medial patellofemoral ligament. Knee 10(3):221-227 
19.

Smith TO, Walker J, Russell N (2007) Outcomes of medial patellofemoral ligament reconstruction for patellar instability: a systematic review. Knee Surg Sports Traumatol Arthrosc 15(11):1301-1314

20.

Steensen RN, Dopirak RM, Maurus PB (2005) A simple technique for reconstruction of the medial patellofemoral ligament using a quadriceps tendon graft. Arthroscopy 21(3):365-370

21.

Steensen RN, Dopirak RM, McDonald WG 3rd (2004) The anatomy and isometry of the medial patellofemoral ligament: implications for reconstruction. Am J Sports Med 32(6):1509-1513

22.

Steiner TM, Torga-Spak R, Teitge RA (2006) Medial patellofemoral ligament reconstruction in patients with lateral patellar instability and trochlear dysplasia. Am J Sports Med 34(8):1254-1261

23.

Tateishi T, Tsuchiya M, Motosugi N, Asahina S, Ikeda H, Cho S, Muneta T (2011) Graft length change and radiographic assessment of femoral drill hole position for medial patellofemoral ligament reconstruction. Knee Surg Sports Traumatol Arthrosc 19(3):400-407

24.

Wijdicks CA, Griffith CJ, LaPrade RF, Johansen S, Sunderland A, Arendt EA, Engebretsen L (2009) Radiographic identification of the primary medial knee structures. J Bone Joint Surg Am 91(3):521-529

25.

Yoo YS, Chang HG, Seo YJ, Byun JC, Lee GK, Im H, Song SY (2012) Changes in the length of the medial patellofemoral ligament: an in vivo analysis using 3 dimensional computed tomography. Am J Sports Med 40(9):2142-2148

26.

Zaffagnini S, Marcheggiani Muccioli GM, Grassi A, Bonanzinga T, Marcacci M (2014) Minimally invasive medial patellofemoral ligament reconstruction with fascia lata allograft: surgical technique. Knee Surg Sports Traumatol Arthrosc 22(10):2426-2430 